\title{
Improved Fast Time Reversible Algorithm and Its Applications to Large-Scale Simulation of Ice
}

\author{
Yasuhiro Kajima* \\ Nagoya Zokei University, Komaki \\ Aichi, 485-8563, Japan \\ *Corresponding author
}

\author{
Shuji Ogata \\ Graduate School of Engineering \\ Nagoya Institute of Technology \\ Nagoya, Aichi 466-8555, Japan
}

\begin{abstract}
A time-reversible algorithm for the rotation of rigid body in the quaternion representation named "Improved Fast Time-reversible algorithm (IFT)" is proposed. In a preceding paper, we have proposed "Fast Time-reversible algorithm (FT)" that is not only time reversible but also conserves the total energy well and one of the simplest time reversible ones. However, the algorithm involves a square root, which yields the risk of computer crash at extreme high temperatures. The IFT gets rid of the risk of crash and the stability is no less than the Fast Timereversible algorithm. The IFT can be used more widely than FT. We also introduce some results concerning quasi liquid layer obtained from large-scale simulations. The simulations were performed by the use of IFT algorithm.
\end{abstract}

Keywords-simulation algorithm; rigid body; rotation; time reversible; molecular dynamics; quasi liquid layer

\section{INTRODUCTION}

The computational power of super computers is rapidly increasing, and recent supercomputers with many nodes enable us to perform large-scale simulation by dividing objects. On the other hand, high-speed intramolecular vibration makes it difficult to take longer time step. However, we can take longer time step by regarding molecules as rigid bodies. In the case of the simulation of water molecules, rigid model potentials such as TIP4P and their variants [1] are known to provide qualitatively correct description of water. Thus water molecules are usually treated as rigid body and several rotational algorithms have been devised and applied to the simulation of water. However, these rotational algorithms seem to be not stable in long runs or somewhat complicated and consume computational power. Therefore, simple and stable rotational algorithm is desired. We have proposed such an algorithm named Fast Time-reversible (FT) algorithm [2]. It is stable and the implementation code is very short. However there is a danger of computer crash at extremely high temperature. It is true that we can avoid such danger by the use of "IF" statement, however, it is desirable to remove such a danger essentially. Thus we propose an improved FT algorithm (IFT) that removes such a danger essentially.

We applied the IFT algorithm to a small droplet and tested its stability. We also applied IFT to large-scale simulations of ice and studied the behavior of the quasi liquid layer (QLL) (c.f. [3]). Here, QLL is a liquid like layer that is widely believed to exist on ices even under the melting point [4]. Since it is considered to play very important roles in many aspects, e.g., in the mechanics of skating and in the formation of acid snow, many studies have been carried out to investigate the property of QLL (e.g., [5-6]). We introduce some novel results obtained from our simulation using IFT and FT algorithm [3].

\section{FT AND IMPROVED FT ALGORITHMS}

\section{A. FT Algorithm}

Here we recall the notion of the FT algorithm briefly. The FT algorithm firstly determines the angular position of each molecule of step- $(n+1)$ by determining its quaternion $\vec{q}_{n+1}$ through the second order Taylor expansion of $\vec{q}$ as usual:

$$
\vec{q}_{n+1}=\vec{Q}\left(\vec{q}_{n}, \vec{\omega}_{n}, \Delta t\right),
$$

where $\Delta t$ is a time step, $\vec{\omega}_{n}$ is the angular velocityand $\vec{q}$ 's are the quaternions, that is, $\vec{q}=\left(q_{1}, q_{2}, q_{3}, q_{4}\right),|\vec{q}|=1$, and by which $3 \times 3$ rotational matrix is represented, and through the matrix we determine the angular position of the molecule. It is known that the representation by means of quaternion is free of singularities.

Next, the angular velocity is determined so as to satisfy the time reversibility condition: $\vec{q}_{n}=\vec{Q}\left(\vec{q}_{n+1}, \vec{\omega}_{n+1},-\Delta t\right)$. In other words,

$$
\vec{q}_{n}=\vec{Q}\left(\vec{Q}\left(\vec{q}_{n}, \vec{\omega}_{n}, \Delta t\right), \vec{\omega}_{n+1},-\Delta t\right) .
$$

Writing down explicitly the equation (1) above, we have:

$$
\vec{q}_{n+1}=\left(\begin{array}{cccc}
d & -u_{x} & -u_{y} & -u_{z} \\
u_{x} & d & u_{z} & -u_{y} \\
u_{y} & -u_{z} & d & u_{x} \\
u_{z} & u_{y} & -u_{x} & d
\end{array}\right) \vec{q}_{n},
$$


where

$$
\vec{u}_{n}=\left(u_{x}, u_{y}, u_{z}\right) \equiv \frac{1}{2} \vec{\phi}_{n}(\Delta t) \Delta t, \quad d=\sqrt{1-\left|\vec{u}_{n}\right|^{2}}
$$

Here, $\vec{\phi}_{n}(\Delta t)$ is a vector obtained by Taylor expansion with respect to $\Delta t$. It depends on $\vec{\omega}_{n}$, torque, angular position, and some constants depending on the rigid body.

Denote the matrix in (3) by $M\left(\vec{u}_{n}\right)$. Then, the time reversibility condition (2) can be written as follows:

$$
\vec{q}_{n}=M\left(-\frac{1}{2} \vec{\phi}_{n+1}(-\Delta t) \Delta t\right) M\left(\frac{1}{2} \vec{\phi}_{n}(\Delta t) \Delta t\right) \vec{q}_{n}
$$

Since the matrix $M$ is orthogonal and satisfies

$$
M(\vec{u})^{T}=M(-\vec{u})
$$

For an arbitrary $u,(5)$ implies

$$
\vec{\phi}_{n+1}(-\Delta t) \Delta t=\vec{\phi}_{n}(\Delta t)
$$

Equation (7) yields algebraic equations with respect to $\vec{\omega}_{n+1}$ whose degree is more than five. It is difficult to solve such equations algebraically, so we have modified (3) slightly as below within the error of the Taylor expansion (i.e., $O\left(\Delta t^{3}\right)$ ) to make (7) combinations of linear equations. The linear equations so obtained can be solved easily and we get the angular velocity at $(n+1)$-step. We note that the time reversibility condition holds completely even after the modification.

Now the modified $\vec{\phi}_{n}(\Delta t)$ is given by the following successive sequence of assignment:

$$
\begin{aligned}
& \phi_{x}(1) \leftarrow \omega_{n, x}, \phi_{y}(1) \leftarrow \omega_{n, y}, \phi_{z}(1) \leftarrow \omega_{n, z}, \\
& \phi_{x}(2) \leftarrow \phi_{x}(1)+\delta t_{x} \Delta t, \\
& \phi_{y}(2) \leftarrow \phi_{y}(1)+\lambda t_{y} \Delta t, \\
& \phi_{z}(2) \leftarrow \phi_{z}(1)+\mu t_{z} \Delta t \\
& \phi_{x} \leftarrow \phi_{x}(2)+\alpha \phi_{y}(2) \phi_{z}(2) \Delta t, \\
& \phi_{y} \leftarrow \phi_{y}(2)+\alpha \phi_{x} \phi_{z}(2) \Delta t, \\
& \phi_{z} \leftarrow \phi_{z}(2)+\alpha \phi_{x} \phi_{y} \Delta t,
\end{aligned}
$$

where $\vec{\phi}=\left(\phi_{x}, \phi_{y}, \phi_{z}\right)$,

$$
\begin{aligned}
& \alpha=\frac{I_{x}-I_{z}}{2 I_{x}}, \quad \beta=\frac{I_{z}-I_{x}}{2 I_{y}}, \quad \gamma=\frac{I_{x}-I_{y}}{2 I_{z}}, \\
& \delta=\frac{1}{2 I_{x}}, \quad \lambda=\frac{1}{2 I_{y}}, \quad \mu=\frac{1}{2 I_{z}},
\end{aligned}
$$

$I_{x}, I_{y}, I_{z}$ are (arbitrarily taken) principal moments of inertia of the rigid body, $\vec{t}=\left(t_{x}, t_{y}, t_{z}\right)$ is a torque, and $\leftarrow$ implies assignment. The error of $\vec{q}_{n+1}$ caused by the modifications above is within $O\left(\Delta t^{3}\right)$, which is same as that of the Taylor expansion. The choices of $I_{x}, I_{y}, I_{z}$ influence the stability of the FT algorithm but only slightly [2].

The modifications of $\vec{\phi}_{n}(\Delta t)$ enable us to solve (7) by proceeding the sequence (8) from the opposite direction to get the angular velocity $\vec{\omega}_{n+1}$ at $(n+1)$-step.

As is seen from the definition of $\vec{u}$ in (4), there is a danger that the number $1-|\vec{u}|^{2}$ inside the square root may become negative when $\Delta t$ is very large or simulation is performed at very high temperature. In our actual simulation of 499 water molecules with $\Delta t=4 \mathrm{fs}$ at about $300 \mathrm{~K}$, we never met such situations. However, when we performed long time simulation of 499 water molecules at more than $2000 \mathrm{~K}$ for testing purposes, $1-|\vec{u}|^{2}$ became negative and the simulation has crashed. Of course we can avoid the crash by using IF statement, however, it is desirable to get rid of such a square root. The square root in (4) is removed in the Improved FT algorithm described below.

\section{B. Improved FT Algorithm}

In the IFT algorithm, the equations given above are slightly modified. We replace $d=\sqrt{1-\left|\vec{u}_{n}\right|^{2}}$ with $d=1-\frac{1}{2}\left|\vec{u}_{n}\right|^{2}$ in the definition of the matrix $M\left(\vec{u}_{n}\right)$ in (3), and then $\vec{q}_{n+1}$ is calculated through (3). Then we add the procedure of normalization of the quaternion $\vec{q}_{n}$ after (3). That is, we add the procedure

$$
\vec{q}_{n+1} \leftarrow \vec{q}_{n+1} /\left|\vec{q}_{n+1}\right|
$$

After (3). It is equivalent to

$$
\vec{q}_{n+1}=\frac{1}{\sqrt[3]{\left|M\left(\vec{u}_{n}\right)\right|}} M\left(\vec{u}_{n}\right) \vec{q}_{n}
$$


We define $\vec{\phi}_{n}(\Delta t)$ by (8) and get $\vec{\omega}_{n+1}$ as in the same way as FT algorithm.

Here we show that the modification preserves the time reversibility. The modified matrix $M(\vec{u})$ above is no longer orthogonal but satisfies the equation

$$
M(\vec{u})^{T} M(\vec{u})=c I,
$$

where $I$ is the identity matrix and $c=1+\frac{1}{4}|\vec{u}|^{2}=(\sqrt[3]{|M(\vec{u})|})^{2}$. Moreover, (6) holds even after the modification. Since (6), (7), and (12) yield

$$
\frac{1}{\sqrt[3]{\left|M\left(\vec{u}_{n}\right)^{T}\right|}} M\left(\vec{u}_{n}\right)^{T} \frac{1}{\sqrt[3]{\left|M\left(\vec{u}_{n}\right)\right|}} M\left(\vec{u}_{n}\right)=I
$$

And

$$
M\left(\vec{u}_{n+1}^{\prime}\right)=M\left(\vec{u}_{n}\right)^{T}
$$

We obtain

$$
\frac{1}{\sqrt[3]{\left|M\left(\vec{u}_{n+1}^{\prime}\right)\right|}} M\left(\vec{u}_{n+1}^{\prime}\right) \frac{1}{\sqrt[3]{\left|M\left(\vec{u}_{n}\right)\right|}} M\left(\vec{u}_{n}\right)=I
$$

where

$$
\vec{u}_{n+1}^{\prime}=\frac{1}{2} \vec{\phi}_{n+1}(-\Delta t)(-\Delta t), \quad \vec{u}_{n}=\frac{1}{2} \vec{\phi}_{n}(\Delta t) \Delta t .
$$

Therefore, we have

$$
\begin{aligned}
& \vec{q}_{n}=\frac{1}{\sqrt[3]{\left|M\left(\vec{u}_{n+1}^{\prime}\right)\right|}} M\left(\vec{u}_{n+1}^{\prime}\right) \frac{1}{\sqrt[3]{\left|M\left(\vec{u}_{n}\right)\right|}} M\left(\vec{u}_{n}\right) \vec{q}_{n} \\
& =\frac{1}{\sqrt[3]{\left|M\left(\vec{u}_{n+1}^{\prime}\right)\right|}} M\left(\vec{u}_{n+1}^{\prime}\right) \vec{q}_{n+1},
\end{aligned}
$$

which implies that the IFT algorithm satisfies time reversibility.

In practice, it is sufficient to calculate updated quaternion by (3) with $d=1-\frac{1}{2}\left|\vec{u}_{n}\right|^{2}$, and normalize the quaternion at every step.

\section{COMPUTATION RESUltS: STABILITY AND} APPLICATIONS TO LARGE-SCALE SimUlations OF ICE

We have applied the Improved FT algorithm to simulations of water and ice. We firstly performed simulations with 499 water molecules at $300 \mathrm{~K}$ to test the stability of IFT, and secondly, we performed large-scale simulation of ice composed of $1,382,400$ water molecules to study their quasi liquid layers.

In our simulations, water molecules were treated as rigid bodies and we employed TIP4P potential. Water droplet and ices are put in a sufficiently large vacuum box. Note that the melting point of TIP4P ice is about $230 \mathrm{~K}$.

In the following, we show the stability of IFT and introduce some of the results obtained through large-scale simulation.

\section{A. Stability of the Improved FT}

Figure 1 shows the global errors for symplectic, FT, and IFT algorithms in simulations of ice with 499 water molecules at $300 \mathrm{~K}$ at several time steps. The global errors are defined by

$$
\varepsilon=\left\langle\left|\frac{E(i+10000)}{E(i)}-1\right|\right\rangle
$$

where $E(i)$ is the total energy of the system at step-i and the average $\langle\cdots\rangle$ is taken over all possible $i$ and runs of $10^{5}$ steps. We quote the global errors for symplectic algorithm from [2] for reference. As is seen from the figure, the global errors in FT algorithm are almost the same as that of IFT algorithm, and inferior to the symplectic algorithm by about an order of magnitude.

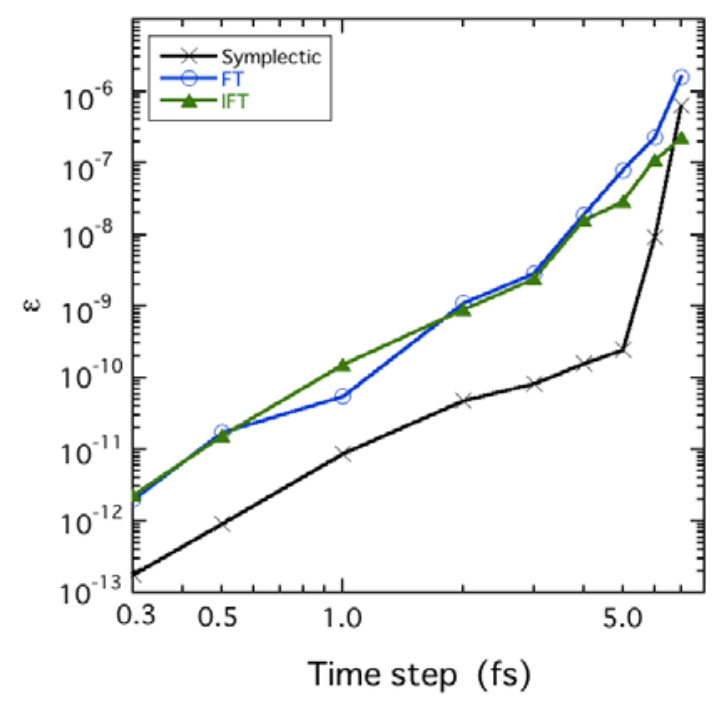

FIGURE I. GLOBAL ERRORS IN THE WATER DROPLET SIMULATION AT T=300 K IN THE SYMPLECTIC, FT, AND IFT ALGORITHMS 


\section{B. Applications to Large-Scale Simulation of Ice}

As mentioned in [2], the FT algorithm has a feature that it consists of fewer operations and thus it can compute rotational motion faster than any other time reversible algorithm. For that reason, we have adopted the FT algorithm in performing large-scale simulations of ice [3]. The number of operations in IFT algorithm is only two more than that of the FT algorithm, and hence the increase of the computational time required to compute rotational motion is about $2 \%$. In addition to that, the computer will not crash even if we heat the simulation system rapidly. Thus we think the IFT algorithm is suitable for largescale simulation.

Here we show briefly simulation results of ice composed of $1,382,400$ water molecules. In the simulation, we adopted fast multipole method to compute the Coulomb forces between distant atoms [8]. Forces between atoms separated less than $8.9 \AA$ are computed directly. Simulations were performed for temperatures $215 \mathrm{~K}, 220 \mathrm{~K}$, and $227 \mathrm{~K}$ with time step=2 fs. As mentioned before, the melting temperature of TIP4P ice is about $230 \mathrm{~K}$. Figure 2 (a) and (b) depicts oblique view and top view of the ice at $220 \mathrm{~K}$ after $5 \mathrm{~ns}$ simulation. As seen from the Figure 2, the surface of the ice is bumpy. The water molecules of the ice is colored with respect to the height in the direction of c-axis, and we set the height of the bottom of the bumpy surface as 0 .

We summarize the results obtained by the simulation. Similar results have been obtained by adopting FT algorithm for a smaller ice [3].

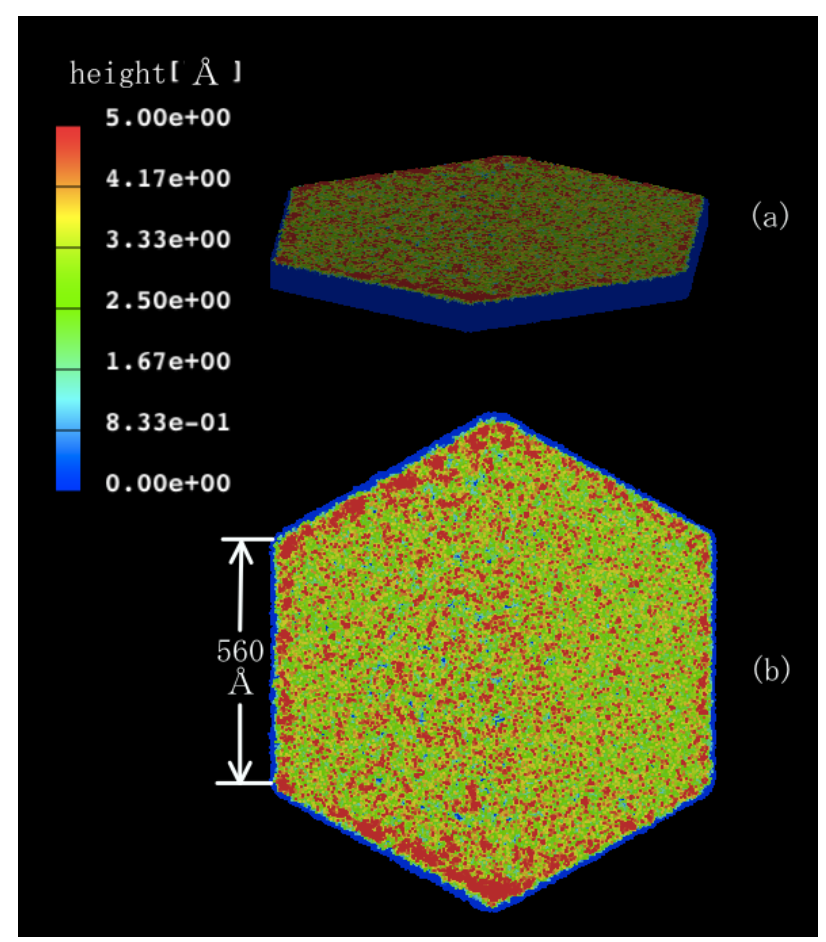

FIGURE II. OBLIQUE VIEW (A) AND TOP VIEW (B) OF THE ICE CRYSTAL AT $220 \mathrm{~K}$ AFTER 5 NS SIMULATION
- The quasi liquid layer is bumpy, and the bumps increase with the rise of temperature, and the average heights of the bumps reach $10 \AA$ slightly below the melting point.

- The liquid bumps fluctuate to form and break at variety of places in a random manner.

- At relatively lower temperatures, the ice molecules melt easily when they are located under the trenches. Moreover, the melted areas newly covered with bumps recrystallize.

\section{CONCLUSION}

We have proposed an improved version of the Fast Timereversible algorithm (IFT). The stability of IFT algorithm was compared with FT algorithm and symplectic algorithm through simulations of water droplet. The global errors of IFT algorithm are almost the same as that of FT algorithm, and inferior to the symplectic algorithm. The computation timing of IFT is almost the same as FT, and IFT is one of the fastest rotational algorithms for rigid body. Moreover the IFT algorithm can be used even at very high temperature, where FT algorithm may crash. The improvement is not a major improvement, however, we believe that it is a significant improvement.

\section{REFERENCES}

[1] C. Vega, J. L. F. Abascal, M. M. Conde, and J. L. Aragones, "What ice can teach us about water interactions: a critical comparison of the performance of different water models" Faraday Discussions, 141, pp. $251,2009$.

[2] Y. Kajima, M. Hiyama, S. Ogata, R. Kobayashi and T. Tamura, "Fast time reversible algorithms for molecular dynamics of rigid-body," J. Chem. Phys., 136, pp. 234105, 2012.

[3] Y. Kajima, S. Ogata, R. Kobayashi, M. Hiyama, and T. Tamura, "Fluctuating Local Recrystallization of Quasi-Liquid Layer of SubMicrometer-Scale Ice: A Molecular Dynamics Study,” J. Phys. Soc. Jpn., 83, pp. 83601, 2014

[4] M. Faraday, "Note on regelation," Proc. R. Soc. London, 10, pp. 440

[5] C. L. Bishop, D. Pan, L. M. Liu, G. A. Tribello, A. Michaelides, E. G. Wang, and B. Slater, "On thin ice: surface order and disorder during premelting," Faraday Discussions, 141, pp. 277, 2009.

[6] K. Bolton, and J. B. C. Petterson, "A Molecular Dynamics Study of the Long-Time Ice Ih Surface Dynamics”, J. Phys. Chem., 104, pp.1590, 2000

[7] D. J. Evans, "On the representation of orientation space," Mol. Phys. 34 pp. $317,1977$.

[8] S. Ogata, T. J. Campbell, R. K. Kalia, A. Nakano, P. Vashishta, and S. Vemparala, "Scalable and portable implementation of the fast multipole method on parallel computers," Comp. Phys. Comm., 153, pp. 445, 2003. 